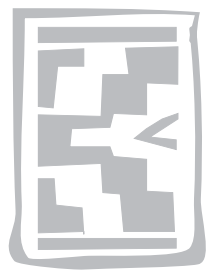

\title{
Toxicity, pathophysiology and pathology in sheep following dosing of the nephrotoxic plant Nolletia gariepina (DC) Mattf.
}

\author{
R.A. MEINTJES ${ }^{1}$, C.J. BOTHA ${ }^{2}$ and L. PROZESKY²
}

\begin{abstract}
MEINTJES, R.A., BOTHA, C.J. \& PROZESKY, L. 2005. Toxicity, pathophysiology and pathology in sheep following dosing of the nephrotoxic plant Nolletia gariepina (DC) Mattf. Onderstepoort Journal of Veterinary Research, 72:39-53

Ingestion of the plant Nolletia gariepina was confirmed as the cause of acute mortalities in cattle in the Kuruman area of the Northern Cape Province of South Africa. The aim of this trial was to investigate the toxic effects of this plant with respect to clinical signs, pathophysiology and pathology using the sheep as a model. At dosages of $1.5 \mathrm{~g}$ dried, milled plant material $/ \mathrm{kg}$ body mass there were no detectable abnormal findings, while at dosages of $2.8-3.0 \mathrm{~g} / \mathrm{kg}$ most of the animals died acutely. In subacutely affected sheep, depression, inappetance, teeth grinding, tachycardia, weak ruminal movements and recumbency were noticed. The most prominent pathophysiological changes observed, included a sharp rise in non-protein nitrogen substances in the plasma, remarkable decline in glomerular filtration rate, increase in sodium and potassium excretion, and a rise in urine gamma glutamyltransferase activity. Macroscopically a severe nephrosis was present in all the animals. The most important findings detected histologically were necrosis of the proximal convoluted tubular epithelium and large numbers of protein casts in the lumens.
\end{abstract}

Keywords: Kidney, nephrotoxicity, Nolletia gariepina, plant poisoning, sheep

\section{INTRODUCTION}

Farmers in the Kuruman district of the Northern Cape Province, Republic of South Africa have recently experienced mortalities in their free-ranging cattle herds. The mortalities, approximately 200 head of cattle, were ascribed to nephrotoxicity induced by a previously unknown poisonous plant (Du Plessis, Joubert, Prozesky, Naudé, Herman \& Van der Westhuizen 2004). Clinically, weakness,

1 Department of Anatomy and Physiology, Faculty of Veterinary Science, University of Pretoria, Private Bag X04, Onderstepoort, 0110 South Africa. E-mail: roy.meintjes@up.ac.za

2 Department of Paraclinical Sciences, Faculty of Veterinary Science, University of Pretoria, Private Bag X04, Onderstepoort, 0110 South Africa. E-mail: christo.botha@up.ac.za and leon.prozesky@up.ac.za

Accepted for publication 27 July 2004-Editor malaise, and perineal and brisket oedema were observed. Necropsy findings included severe anasarca, ascites, perirenal oedema and nephrosis, and mild hepatosis. Microscopical examination revealed extensive degeneration and necrosis of tubular epithelial cells in the renal cortex, accompanied by large numbers of protein and, sometimes, cellular casts in the tubular lumens. Dosing trials conducted by the Division of Toxicology, Onderstepoort Veterinary Institute confirmed Nolletia gariepina (DC) Mattf. as the causative plant (Du Plessis 2004).

The primary aim of the current trial was to elucidate the pathophysiological changes in renal function in sheep induced by N. gariepina. A secondary objective was to evaluate the toxic effects and pathological findings following administration of this nephrotoxic plant to sheep. 


\section{MATERIALS AND METHODS}

Nolletia gariepina was collected on the farm Tom Brown, situated in the quadrant $2226 \mathrm{Cb}$ of the Kuruman district, Northern Cape. The plant material was air dried, finely milled and frozen (at $-10^{\circ} \mathrm{C}$ ) before being used.

Nine Mutton Merino wethers, 9-18 months of age, were individually placed in steel metabolic crates in an experimental animal housing facility. The room temperature was set and maintained at $22^{\circ} \mathrm{C}$. Lucerne hay and drinking water were available ad lib. To facilitate the collection of unsoiled urine the sheep were fitted with faecal collection bags. The total volume of urine voided per day was collected in refrigerated containers located under each crate.

The sheep were placed in the metabolic crates for an adjustment period of 5 days prior to a 5-day control phase during which baseline values for the different parameters were established as each animal served as its own control. Immediately following the control period the sheep were deprived of feed for $24 \mathrm{~h}$ and then dosed with dried, milled plant material (1.5-3 $\mathrm{g} / \mathrm{kg}$ body mass) by stomach tube as indicated in Table 1.

Daily clinical examinations (habitus, rectal temperature, pulse, respiration and rumen motility) were conducted to evaluate the animals' health. Feed and water intake and urine volume were recorded daily.

An aliquot of urine was collected for conventional urinalysis (Combur urinary dip stick [Merck]). Urinary creatinine, sodium (Na) and potassium (K) concentrations, gamma glutamyltransferase (GGT) activity and osmolarity were also determined. The GGT:creatinine ratios served as early detection of renal tubular damage.

Blood samples were collected for determination of haematocrit, osmolarity and plasma protein, $\mathrm{Na}, \mathrm{K}$, creatinine and urea concentrations. In addition, GGT and aspartate aminotransferase (AST) activities were determined to establish if any hepatic damage had occurred.

From the above variables the following were derived:

\section{Glomerular filtration rate (GFR)}

The plasma clearance of endogenous creatinine was equated to GFR on the assumption that creatinine is neither secreted nor absorbed by the renal tubule in sheep (Nawaz \& Shah 1984; Bastl, Rudnick \& Nairns 1985).

$$
\begin{aligned}
& \text { Thus, GFR }=U_{\text {vol }} \cdot U_{\text {[creatinine] }} / \mathrm{PI}_{\text {[creatinine] }}(\ell / \mathrm{d}) \\
& \text { where } \\
& \mathrm{U}_{\mathrm{vol}} \quad=\underset{(\ell / \mathrm{d})}{\text { Volume of urine passed per day }} \\
& \mathrm{U}_{\text {[creatinine] }}=\underset{\text { (mMolar) }}{\text { Creatinine concentration in urine }} \\
& \mathrm{PI}_{[\text {creatinine] }}=\underset{\mathrm{ma} \text { (mMolar) }}{\text { Creatinine concentration in plas- }}
\end{aligned}
$$

\section{Fractional excretion of sodium $\left(\mathrm{FE}_{\mathrm{Na}}\right)$}

(Bastl et al. 1985)

This represents the amount of filtered sodium excreted in the urine expressed as a percentage.

$$
\begin{aligned}
\mathrm{FE}_{\mathrm{Na}} & =U_{\mathrm{vol}} \cdot U_{[\mathrm{Na}]} \cdot 100 / \mathrm{GFR} \cdot \mathrm{PI}_{[\mathrm{Na}]}(\%) \\
\text { where } & = \\
\mathrm{PI}_{[\mathrm{Na}]}= & \begin{array}{l}
\text { Sodium concentration in plasma } \\
\text { (mMolar) }
\end{array} \\
\mathrm{U}_{[\mathrm{Na}]}= & \begin{array}{l}
\text { Sodium concentration in urine } \\
\text { (mMolar) }
\end{array}
\end{aligned}
$$

The carcasses of sheep that died or were euthanased (overdose of pentobarbitone sodium [Euthanaze, Centaur] administered intravenously) were submitted for necropsy. Tissue specimens (approximately $10 \mathrm{~mm}^{3}$ ) from several organs were collected and fixed by immersion in $10 \%$ neutral buffered formalin for at least 5-7 days. Specimens were routinely processed for light microscopy and stained with haematoxylin and eosin (HE).

Renal tissue specimens were collected from two cases (no. 13 and 42 ) within minutes of death, cut into $1 \mathrm{~mm}^{2}$ blocks and fixed in labelled glass vials containing $2.5 \%$ gluteraldehyde in $4 \% 0.1 \mathrm{M}$ Millonig's buffer. Samples were routinely processed for electron microscopy using a transmission electron microscope and photographed.

\section{RESULTS}

\section{Toxicity}

The clinical signs observed and pathological findings are summarized in Table 1. Four of seven animals that were dosed with $3 \mathrm{~g} / \mathrm{kg} \mathrm{N}$. gariepina plant material died acutely within $24 \mathrm{~h}$. One sheep, which was depressed, recumbent and exhibited respiratory distress (hyperventilation, groaning, frothing at the mouth) was euthanased $8 \mathrm{~h}$ after being dosed with $3 \mathrm{~g} / \mathrm{kg}$ milled plant material. Only two animals, which had received $3 \mathrm{~g} / \mathrm{kg}$ plant material, survived 
R.A. MEINTJES, C.J. BOTHA \& L. PROZESKY

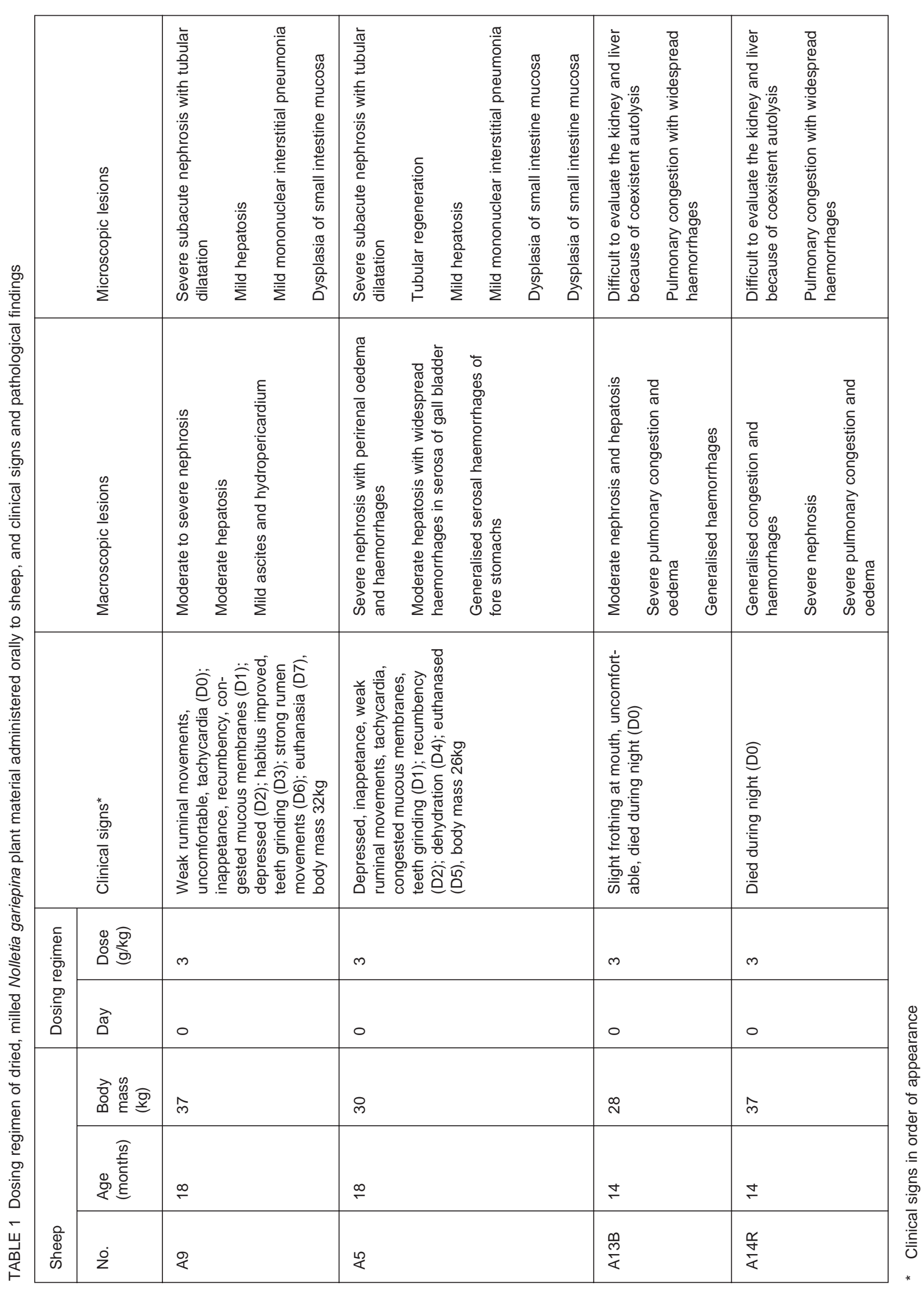




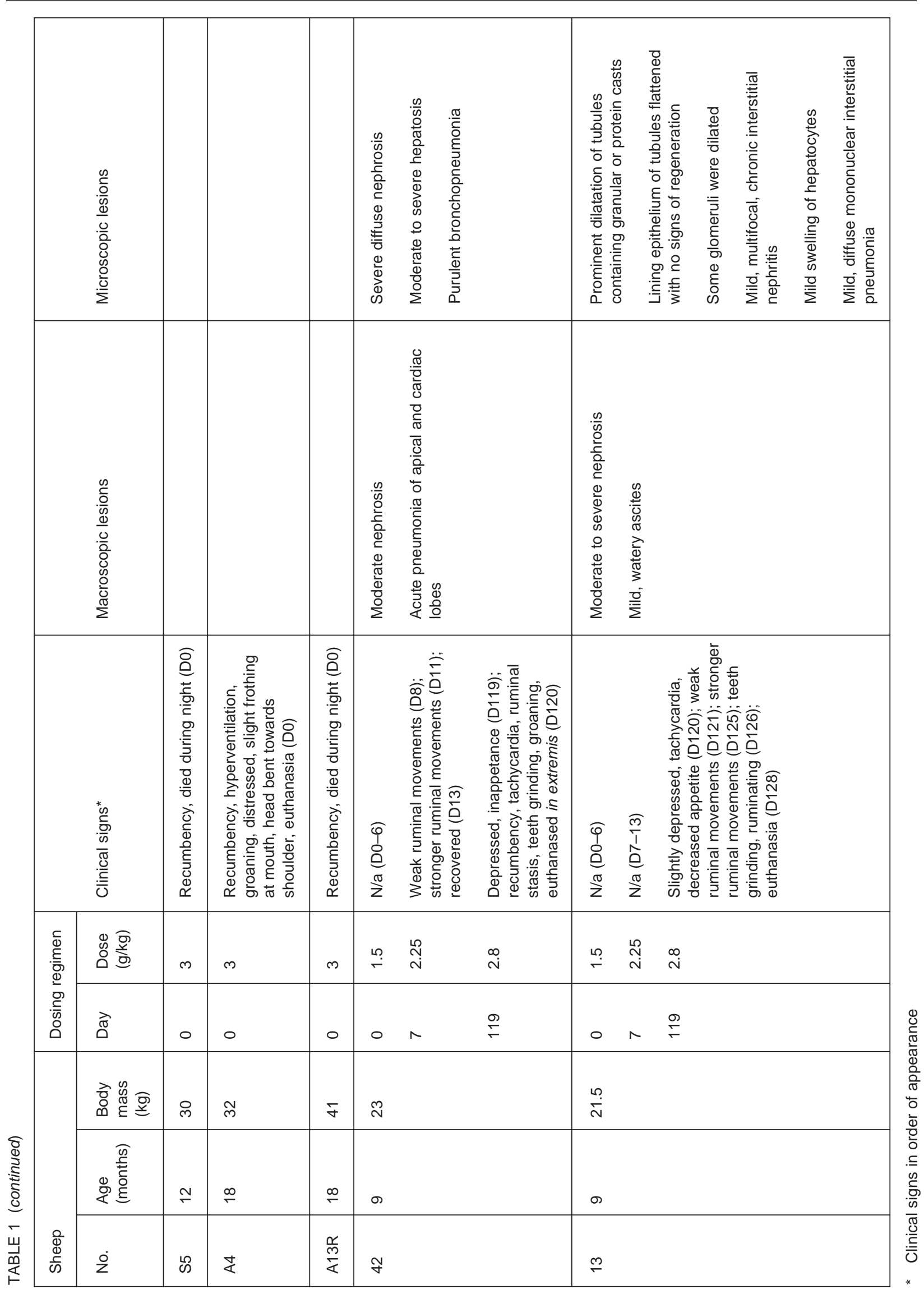




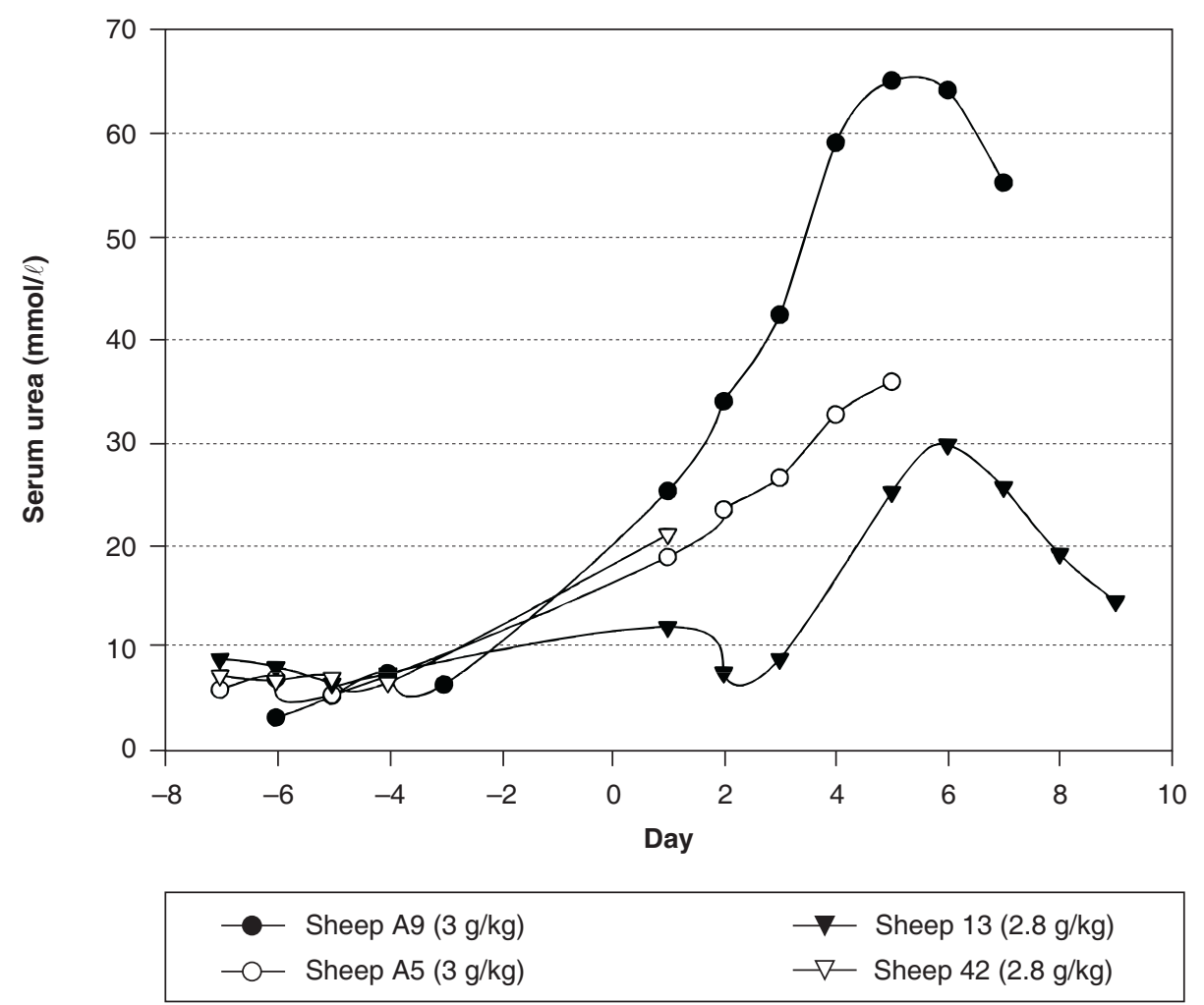

FIG. 1 Serum urea concentration in four sub-acutely affected sheep following administration of dried, milled Nolletia gariepina plant material

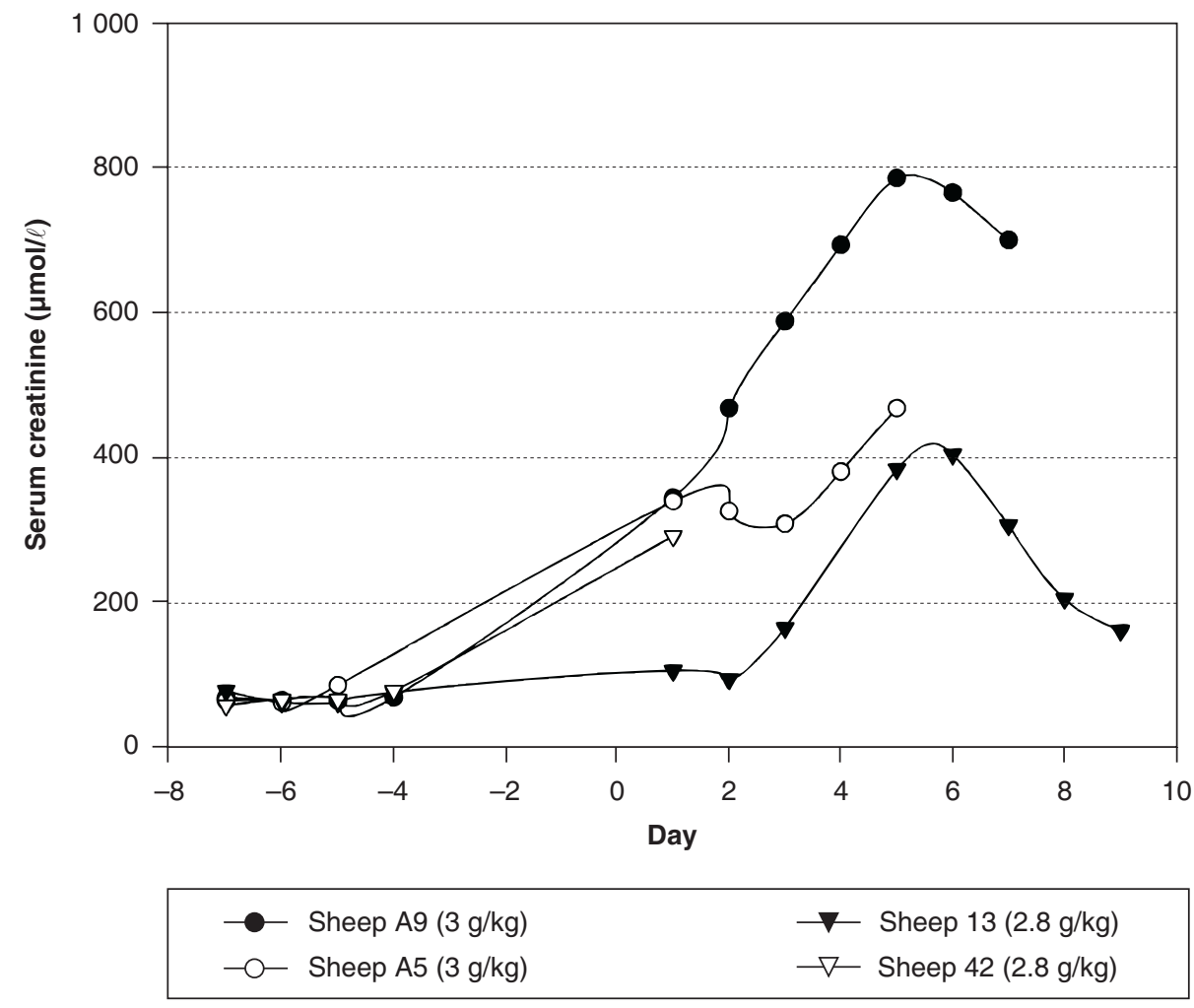

FIG. 2 Serum creatinine concentration in four sub-acutely affected sheep following administration of dried, milled Nolletia gariepina plant material 
for longer periods, but were depressed, anorexic and had weak rumen movements and tachycardia.

Administration of half the dose $(1.5 \mathrm{~g} / \mathrm{kg})$ to two wethers elicited no clinical effects. Following a washout period of 7 days the same two sheep were dosed with $2.25 \mathrm{~g} / \mathrm{kg} \mathrm{N}$. gariepina plant material. Only one animal was mildly affected clinically (decreased appetite and weak ruminal movements), but was judged fully recovered after 6 days. More than 3 months later the same two wethers were dosed again with $2.8 \mathrm{~g} / \mathrm{kg}$ and at this dose, moderate to severe clinical signs developed (Table 1).

\section{Pathophysiology}

\section{Non-protein nitrogen (NPN) substances in serum}

The most noticeable aberrations in blood chemistry were the remarkable increases in serum urea and creatinine concentrations in sheep that survived for $24 \mathrm{~h}$ or longer (Fig. 1 and 2).

\section{Serum AST and GGT activities}

A moderate increase in AST activity (96-157 U/l) above the reference range $(5-60 \mathrm{U} / \ell)$ occurred in some sheep. There was no noticeable increase in GGT activity.

\section{Daily urine volumes}

Following dosage of the sheep with $N$. gariepina, even at doses of $1.5 \mathrm{~g} / \mathrm{kg}$, urine production in gen- eral increased. In some cases, however, e.g. sheep A5 and sheep A9 dosed with $3 \mathrm{~g} / \mathrm{kg}$, the increase was preceded by virtual anuria on the day immediately following dosage (Table 2).

\section{Urinalysis}

Semi-quantitative urinalysis (urinary dip stick) revealed mild proteinuria and presence of haemoglobin in the urine after dosing.

\section{Glomerular filtration rate (GFR)}

Dramatic decreases in GFR were calculated in sheep, which survived at least one day post-dosing, when the plant material was dosed in excess of $1.5 \mathrm{~g} / \mathrm{kg}$ (Table 3).

\section{Urinary sodium excretion}

During the control phase no sodium could be detected in the urine, but within $24 \mathrm{~h}$ of dosing the Nolletia plant material at doses of $2.8 \mathrm{~g} / \mathrm{kg}$ and above, urinary sodium concentrations rose. The fractional excretion of sodium is given in Table 4.

\section{Urinary potassium excretion}

The fractional excretion of potassium increased, even after receiving relatively low doses $(1.5 \mathrm{~g} / \mathrm{kg})$ of Nolletia plant material. As potassium is excreted by the nephron tubule, values in excess of $100 \%$ are encountered, sometimes even under control conditions.

TABLE 2 Urine volumes $(\ell)$ of sheep-control values and values following dosing with Nolletia gariepina

\begin{tabular}{|c|c|c|c|c|c|c|c|c|c|}
\hline Dose rate & $\begin{array}{l}\text { Sheep } \\
\text { no. }\end{array}$ & $\begin{array}{l}\text { Control } \\
\text { mean (SD) }\end{array}$ & $\begin{array}{l}\text { Day } 1 \\
\text { post } \\
\text { dosing }\end{array}$ & $\begin{array}{l}\text { Day } 2 \\
\text { post } \\
\text { dosing }\end{array}$ & $\begin{array}{l}\text { Day } 3 \\
\text { post } \\
\text { dosing }\end{array}$ & $\begin{array}{l}\text { Day } 4 \\
\text { post } \\
\text { dosing }\end{array}$ & $\begin{array}{l}\text { Day } 5 \\
\text { post } \\
\text { dosing }\end{array}$ & $\begin{array}{l}\text { Day } 6 \\
\text { post } \\
\text { dosing }\end{array}$ & $\begin{array}{l}\text { Day } 7 \\
\text { post } \\
\text { dosing }\end{array}$ \\
\hline $1.5 \mathrm{~g} / \mathrm{kg}$ & $\begin{array}{l}42 \\
13\end{array}$ & $\begin{array}{ll}0.21 & (0.06) \\
0.275 & (0.069)\end{array}$ & $\begin{array}{l}0.64 \\
0.35\end{array}$ & $\begin{array}{l}0.66 \\
0.33\end{array}$ & $\begin{array}{l}0.71 \\
0.27\end{array}$ & & & & \\
\hline $2.25 \mathrm{~g} / \mathrm{kg}$ & $\begin{array}{l}42 \\
13\end{array}$ & $\begin{array}{ll}0.21 & (0.06) \\
0.275 & (0.69)\end{array}$ & $\begin{array}{l}0.64 \\
0.71\end{array}$ & $\begin{array}{l}1.42 \\
0.37\end{array}$ & $\begin{array}{l}3.047 \\
0.445\end{array}$ & $\begin{array}{l}1.7 \\
0.44\end{array}$ & & $\begin{array}{l}2.129 \\
0.52\end{array}$ & $\begin{array}{l}2.042 \\
0.56\end{array}$ \\
\hline $2.8 \mathrm{~g} / \mathrm{kg}$ & $\begin{array}{l}42 \\
13\end{array}$ & $\begin{array}{ll}0.33 & (0.11) \\
0.161 & (0.044)\end{array}$ & $\begin{array}{l}\text { Died } \\
0.59\end{array}$ & 0.28 & 0.64 & 0.71 & 0.6 & 0.6 & 1 \\
\hline $3.0 \mathrm{~g} / \mathrm{kg}$ & $\begin{array}{l}\text { A9 } \\
\text { A5 } \\
\text { A4 } \\
\text { S5 } \\
\text { A14R } \\
\text { A13B } \\
\text { A13R }\end{array}$ & $\begin{array}{ll}0.874 & (0.25) \\
0.643 & (0.165) \\
0.868 & (0.207) \\
0.32 & (0.15) \\
0.573 & (0.14) \\
0.378 & (0.15) \\
0.754 & (0.1)\end{array}$ & $\begin{array}{l}0.099 \\
0 \\
\text { Euth.* } \\
\text { Died } \\
\text { Died } \\
\text { Died } \\
\text { Died }\end{array}$ & $\begin{array}{l}1.37 \\
0.86\end{array}$ & $\begin{array}{l}1.46 \\
3.12\end{array}$ & $\begin{array}{l}1.068 \\
1.21\end{array}$ & $\begin{array}{l}1.305 \\
0.66\end{array}$ & $\begin{array}{l}1.46 \\
\text { Euth* }\end{array}$ & 2.67 \\
\hline
\end{tabular}

* Euth. = Euthanasia 
R.A. MEINTJES, C.J. BOTHA \& L. PROZESKY

TABLE 3 Glomerular filtration rates ( $/$ /day) of sheep—control values and values following dosing with Nolletia gariepina

\begin{tabular}{|c|c|c|c|c|c|c|c|c|c|}
\hline Dose rate & $\begin{array}{l}\text { Sheep } \\
\text { no. }\end{array}$ & $\begin{array}{l}\text { Control } \\
\text { mean (SD) }\end{array}$ & $\begin{array}{l}\text { Day } 1 \\
\text { post } \\
\text { dosing }\end{array}$ & $\begin{array}{l}\text { Day } 2 \\
\text { post } \\
\text { dosing }\end{array}$ & $\begin{array}{l}\text { Day } 3 \\
\text { post } \\
\text { dosing }\end{array}$ & $\begin{array}{l}\text { Day } 4 \\
\text { post } \\
\text { dosing }\end{array}$ & $\begin{array}{l}\text { Day } 5 \\
\text { post } \\
\text { dosing }\end{array}$ & $\begin{array}{l}\text { Day } 6 \\
\text { post } \\
\text { dosing }\end{array}$ & $\begin{array}{l}\text { Day } 7 \\
\text { post } \\
\text { dosing }\end{array}$ \\
\hline $1.5 \mathrm{~g} / \mathrm{kg}$ & $\begin{array}{l}42 \\
13\end{array}$ & $\begin{array}{ll}37.29 & (4.06) \\
49.07 & (8.25)\end{array}$ & $\begin{array}{l}43.05 \\
46.83\end{array}$ & & $\begin{array}{l}44.43 \\
38.33\end{array}$ & & & & \\
\hline $2.25 \mathrm{~g} / \mathrm{kg}$ & $\begin{array}{l}42 \\
13\end{array}$ & $\begin{array}{ll}37.29 & (4.06) \\
49.07 & (8.25)\end{array}$ & $\begin{array}{l}4.8 \\
15.42\end{array}$ & $\begin{array}{l}6.56 \\
26.38\end{array}$ & & $\begin{array}{l}1.93 \\
23.85\end{array}$ & & $\begin{array}{l}3.07 \\
37.44\end{array}$ & \\
\hline $2.8 \mathrm{~g} / \mathrm{kg}$ & $\begin{array}{l}42 \\
13\end{array}$ & $\begin{array}{ll}37.73 & (8.09) \\
33.11 & (9.48)\end{array}$ & $\begin{array}{l}\text { Died } \\
16.53\end{array}$ & 28.06 & 10.71 & & 1.62 & 2.18 & 4.01 \\
\hline $3.0 \mathrm{~g} / \mathrm{kg}$ & $\begin{array}{l}\text { A9 } \\
\text { A5 } \\
\text { A4 } \\
\text { S5 } \\
\text { A14R } \\
\text { A13B } \\
\text { A13R }\end{array}$ & $\begin{array}{ll}87.79 & (11.95) \\
62.03 & (15.15) \\
41.89 & (2.06) \\
75.32 & (1.96) \\
68.14 & (1.55) \\
33.22 & (10.55) \\
103.41 & (12.19)\end{array}$ & $\begin{array}{l}0.49 \\
-a \\
\text { Euth.* } \\
\text { Died } \\
\text { Died } \\
\text { Died } \\
\text { Died }\end{array}$ & $\begin{array}{l}5.27 \\
8.74\end{array}$ & $\begin{array}{l}2.76 \\
15.99\end{array}$ & $\begin{array}{l}1.15 \\
4.65\end{array}$ & $\begin{array}{l}1.59 \\
1.95\end{array}$ & $\begin{array}{l}1.89 \\
\text { Euth*}\end{array}$ & 4.73 \\
\hline
\end{tabular}

* Euth. $=$ Euthanasia

a $\quad=$ No urine produced

TABLE 4 Fractional excretion of sodium (\%) and potassium (\%) in sheep—control values and values following dosing with Nolletia gariepina

\begin{tabular}{|c|c|c|c|c|c|c|c|c|c|c|}
\hline Dose rate & Sheep nc & & $\begin{array}{l}\text { Control } \\
\text { mean } \\
(\mathrm{SD})\end{array}$ & $\begin{array}{l}\text { Day } 1 \\
\text { post } \\
\text { dosing }\end{array}$ & $\begin{array}{l}\text { Day } 2 \\
\text { post } \\
\text { dosing }\end{array}$ & $\begin{array}{l}\text { Day } 3 \\
\text { post } \\
\text { dosing }\end{array}$ & $\begin{array}{l}\text { Day } 4 \\
\text { post } \\
\text { dosing }\end{array}$ & $\begin{array}{l}\text { Day } 5 \\
\text { post } \\
\text { dosing }\end{array}$ & $\begin{array}{l}\text { Day } 6 \\
\text { post } \\
\text { dosing }\end{array}$ & $\begin{array}{l}\text { Day } 7 \\
\text { post } \\
\text { dosing }\end{array}$ \\
\hline $1.5 \mathrm{~g} / \mathrm{kg}$ & $\begin{array}{ll}42 & \mathrm{~N} \\
& \mathrm{~K} \\
13 & \mathrm{~N} \\
& \mathrm{~K}\end{array}$ & $\begin{array}{l}\mathrm{Na} \\
\mathrm{K} \\
\mathrm{Na} \\
\mathrm{K}\end{array}$ & $\begin{array}{ll}0 & \\
82 & (4.2) \\
0 & \\
101 & (11)\end{array}$ & $\begin{array}{l}0 \\
101 \\
0 \\
101\end{array}$ & & $\begin{array}{l}0 \\
195 \\
0 \\
150\end{array}$ & & & & \\
\hline $2.25 \mathrm{~g} / \mathrm{kg}$ & $\begin{array}{ll}42 & \mathrm{~N} \\
& \mathrm{~K} \\
13 & \mathrm{~N} \\
& \mathrm{~K}\end{array}$ & $\begin{array}{l}\mathrm{Na} \\
\mathrm{K} \\
\mathrm{Na} \\
\mathrm{K}\end{array}$ & $\begin{array}{ll}0 & \\
82 & (4.2) \\
0 & \\
101 & (11)\end{array}$ & $\begin{array}{l}0 \\
444 \\
0 \\
180\end{array}$ & $\begin{array}{l}0 \\
695 \\
0 \\
178\end{array}$ & & $\begin{array}{l}0 \\
2533 \\
0 \\
276\end{array}$ & & $\begin{array}{l}0 \\
1989 \\
0 \\
229\end{array}$ & \\
\hline $2.8 \mathrm{~g} / \mathrm{kg}$ & $\begin{array}{ll}42 & \mathrm{~N} \\
& \mathrm{~K} \\
13 & \mathrm{~N} \\
& \mathrm{~K}\end{array}$ & $\begin{array}{l}\mathrm{Na} \\
\mathrm{K} \\
\mathrm{Na} \\
\mathrm{K}\end{array}$ & $\begin{array}{ll}0 & \\
51 & (18) \\
0 & \\
37 & (14)\end{array}$ & $\begin{array}{l}\text { Died } \\
1 \\
33\end{array}$ & $\begin{array}{l}0.1 \\
45\end{array}$ & $\begin{array}{l}5.4 \\
166\end{array}$ & & $\begin{array}{l}16 \\
614\end{array}$ & $\begin{array}{l}7.8 \\
573\end{array}$ & $\begin{array}{l}9.0 \\
378\end{array}$ \\
\hline $3.0 \mathrm{~g} / \mathrm{kg}$ & $\begin{array}{ll}\text { A9 } & \mathrm{N} \\
& \mathrm{K} \\
\text { A5 } & \mathrm{N} \\
& \mathrm{K} \\
\text { A4 } & \mathrm{N} \\
& \mathrm{K} \\
\text { S5 } & \mathrm{N} \\
& \mathrm{K} \\
\text { A14R } & \mathrm{N} \\
& \mathrm{K} \\
\text { A13B } & \mathrm{N} \\
& \mathrm{K} \\
\text { A13R } & \mathrm{N} \\
& \mathrm{K}\end{array}$ & $\begin{array}{l}\mathrm{Na} \\
\mathrm{K} \\
\mathrm{Na} \\
\mathrm{K} \\
\mathrm{Na} \\
\mathrm{K} \\
\mathrm{Na} \\
\mathrm{K} \\
\mathrm{Na} \\
\mathrm{K} \\
\mathrm{Na} \\
\mathrm{K} \\
\mathrm{Na} \\
\mathrm{K}\end{array}$ & $\begin{array}{ll}0 & \\
97 & (53) \\
0 & \\
89 & (15) \\
0 & \\
137 & (39) \\
0 & \\
89 & (38) \\
0 & \\
102 & (20) \\
0 & \\
124 & (46) \\
0 & \\
68 & (8)\end{array}$ & $\begin{array}{l}5.7 \\
315 \\
-^{a} \\
-^{a} \\
\text { Euth.* } \\
\text { Died } \\
\text { Died } \\
\text { Died } \\
\text { Died }\end{array}$ & $\begin{array}{l}1.8 \\
845 \\
0 \\
45\end{array}$ & $\begin{array}{l}11.8 \\
1028 \\
5.9 \\
533\end{array}$ & $\begin{array}{l}13.9 \\
1225 \\
10.1 \\
673\end{array}$ & $\begin{array}{l}11.9 \\
1084 \\
5.25 \\
648\end{array}$ & $\begin{array}{l}16.5 \\
796 \\
\text { Euth* }\end{array}$ & \\
\hline
\end{tabular}

* Euth. = Euthanasia

a $\quad=$ No urine produced 
Toxicity, pathophysiology and pathology of the nephrotoxic plant Nolletia gariepina (DC) Mattf.

TABLE 5 Urine osmolarity (mOsm/ $\ell$ ) of sheep—control values and values following dosing with Nolletia gariepina

\begin{tabular}{|c|c|c|c|c|c|c|c|c|c|}
\hline Dose rate & $\begin{array}{l}\text { Sheep } \\
\text { no. }\end{array}$ & $\begin{array}{l}\text { Control } \\
\text { mean (SD) }\end{array}$ & $\begin{array}{l}\text { Day } 1 \\
\text { post } \\
\text { dosing }\end{array}$ & $\begin{array}{l}\text { Day } 2 \\
\text { post } \\
\text { dosing }\end{array}$ & $\begin{array}{l}\text { Day } 3 \\
\text { post } \\
\text { dosing }\end{array}$ & $\begin{array}{l}\text { Day } 4 \\
\text { post } \\
\text { dosing }\end{array}$ & $\begin{array}{l}\text { Day } 5 \\
\text { post } \\
\text { dosing }\end{array}$ & $\begin{array}{l}\text { Day } 6 \\
\text { post } \\
\text { dosing }\end{array}$ & $\begin{array}{l}\text { Day } 7 \\
\text { post } \\
\text { dosing }\end{array}$ \\
\hline $1.5 \mathrm{~g} / \mathrm{kg}$ & $\begin{array}{l}42 \\
13\end{array}$ & $\begin{array}{ll}2516 & (357) \\
2647 & (79)\end{array}$ & $\begin{array}{l}1267 \\
2312\end{array}$ & & $\begin{array}{l}1721 \\
2900\end{array}$ & & & & \\
\hline $2.25 \mathrm{~g} / \mathrm{kg}$ & $\begin{array}{l}42 \\
13\end{array}$ & $\begin{array}{ll}2516 & (357) \\
2647 & (79)\end{array}$ & $\begin{array}{l}624 \\
813\end{array}$ & $\begin{array}{l}566 \\
1877\end{array}$ & $\begin{array}{l}496 \\
2182\end{array}$ & $\begin{array}{l}566 \\
2237\end{array}$ & & $\begin{array}{l}634 \\
2440\end{array}$ & \\
\hline $2.8 \mathrm{~g} / \mathrm{kg}$ & $\begin{array}{l}42 \\
13\end{array}$ & $\begin{array}{l}1452(222) \\
2355(163)\end{array}$ & Died & 1311 & 990 & 658 & 567 & 659 & 635 \\
\hline $3.0 \mathrm{~g} / \mathrm{kg}$ & $\begin{array}{l}\text { A9 } \\
\text { A5 } \\
\text { A4 } \\
\text { S5 } \\
\text { A14R } \\
\text { A13B } \\
\text { A13R }\end{array}$ & $\begin{array}{lll}1375 & (364) \\
1470 & (597) \\
1071 & (145) \\
1948 & (456) \\
1875 & (406) \\
1947 & (366) \\
1498 & (243)\end{array}$ & $\begin{array}{l}582 \\
\text {-a }^{2} \\
\text { Euth.* } \\
\text { Died } \\
\text { Died } \\
\text { Died } \\
\text { Died }\end{array}$ & $\begin{array}{l}508 \\
493\end{array}$ & $\begin{array}{l}520 \\
255\end{array}$ & $\begin{array}{l}550 \\
539\end{array}$ & $\begin{array}{l}613 \\
567\end{array}$ & $\begin{array}{l}584 \\
\text { Euth.* }\end{array}$ & 444 \\
\hline
\end{tabular}

* Euth. = Euthanasia

a $\quad=$ No urine produced

TABLE 6 Urine gamma glutamyltransferase (GGT) concentrations (units/ $\ell$ ) in sheep—control values and values following dosing with Nolletia gariepina

\begin{tabular}{|c|c|c|c|c|c|c|c|c|c|}
\hline Dose rate & $\begin{array}{l}\text { Sheep } \\
\text { no. }\end{array}$ & $\begin{array}{l}\text { Control } \\
\text { mean (SD) }\end{array}$ & $\begin{array}{l}\text { Day } 1 \\
\text { post } \\
\text { dosing }\end{array}$ & $\begin{array}{l}\text { Day } 2 \\
\text { post } \\
\text { dosing }\end{array}$ & $\begin{array}{l}\text { Day } 3 \\
\text { post } \\
\text { dosing }\end{array}$ & $\begin{array}{l}\text { Day } 4 \\
\text { post } \\
\text { dosing }\end{array}$ & $\begin{array}{l}\text { Day } 5 \\
\text { post } \\
\text { dosing }\end{array}$ & $\begin{array}{l}\text { Day } 6 \\
\text { post } \\
\text { dosing }\end{array}$ & $\begin{array}{l}\text { Day } 7 \\
\text { post } \\
\text { dosing }\end{array}$ \\
\hline $1.5 \mathrm{~g} / \mathrm{kg}$ & $\begin{array}{l}42 \\
13\end{array}$ & $\begin{array}{ll}25 & (13) \\
26 & (8)\end{array}$ & $\begin{array}{l}10 \\
15\end{array}$ & & $\begin{array}{l}10 \\
15\end{array}$ & & & & \\
\hline $2.25 \mathrm{~g} / \mathrm{kg}$ & $\begin{array}{l}42 \\
13\end{array}$ & $\begin{array}{ll}25 & (13) \\
26 & (8)\end{array}$ & $\begin{array}{l}36 \\
9\end{array}$ & $\begin{array}{l}75 \\
36\end{array}$ & & $\begin{array}{l}30 \\
24\end{array}$ & & $\begin{array}{l}18 \\
9\end{array}$ & \\
\hline $2.8 \mathrm{~g} / \mathrm{kg}$ & $\begin{array}{l}42 \\
13\end{array}$ & $\begin{array}{ll}14 & (7) \\
35 & (23)\end{array}$ & $\begin{array}{l}\text { Died } \\
9\end{array}$ & 48 & 117 & 69 & 30 & 39 & 21 \\
\hline $3.0 \mathrm{~g} / \mathrm{kg}$ & $\begin{array}{l}\text { A9 } \\
\text { A5 } \\
\text { A4 } \\
\text { S5 } \\
\text { A14R } \\
\text { A13B } \\
\text { A13R }\end{array}$ & $\begin{array}{ll}10.5 & (1) \\
13 & (3) \\
18 & (15) \\
16 & (7) \\
19 & (6) \\
12 & (3) \\
16 & (2)\end{array}$ & $\begin{array}{l}66 \\
\text {-a }^{2} \\
\text { Euth.* } \\
\text { Died } \\
\text { Died } \\
\text { Died } \\
\text { Died }\end{array}$ & $\begin{array}{l}165 \\
96\end{array}$ & $\begin{array}{l}90 \\
71\end{array}$ & $\begin{array}{l}42 \\
71\end{array}$ & $\begin{array}{l}36 \\
58\end{array}$ & $\begin{array}{l}21 \\
\text { Euth.* }\end{array}$ & 10 \\
\hline
\end{tabular}

* Euth. = Euthanasia

a $\quad$ No urine produced

\section{Urine osmolarity}

In most of the animals, which did not die within $24 \mathrm{~h}$ of dosing the plant material, urine osmolarity decreased markedly (Table 5).

\section{Urinary GGT activity}

Although no change in serum GGT activity was noticed following dosing with Nolletia plant material, urine GGT activity tended to rise and then fall within a week (Table 6).

\section{Pathology}

\section{Macroscopic pathology}

A severe nephrosis was present in all the animals. The kidneys exhibited various degrees of swelling, bulged slightly on cut surface and ranged in colour 

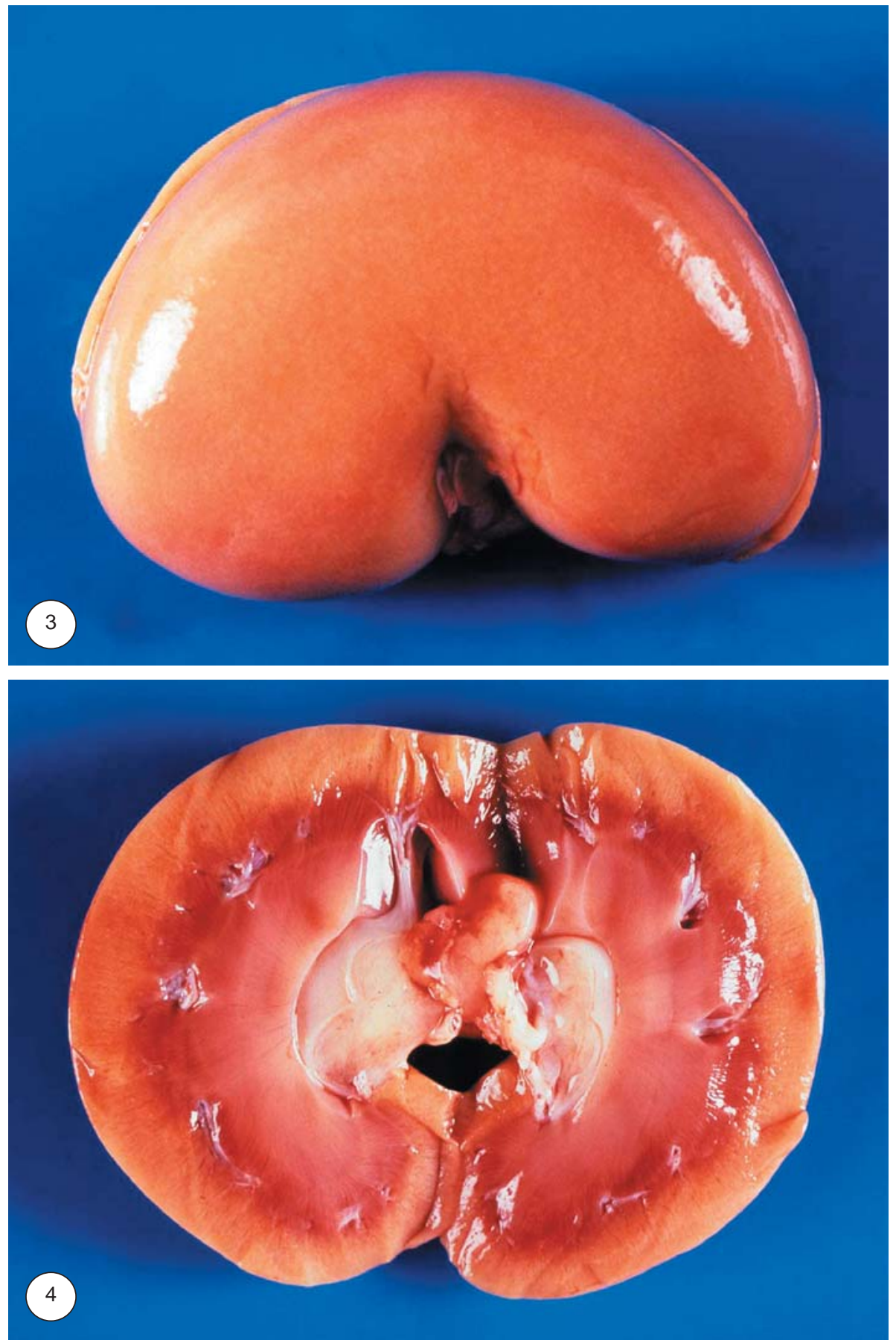

FIG. 3 and 4 Severe nephrosis in a sheep dosed with Nolletia gariepina plant material

from light brown to greyish-white (Fig. 3 and 4). Widespread serosal haemorrhages; mild accumulation of fluid in the body cavities; moderate hepatosis characterized by mild swelling of the liver with rounded edges, slightly pale colour and a taut capsule; and lung congestion and oedema were often seen (Table 1).

\section{Light microscopy}

KIDNEY

A severe nephrosis was the most striking lesion present. The epithelium of the proximal convoluted tubules (PCT) in the cortex and straight tubules in the cortico-medullary junction was the most severe- 

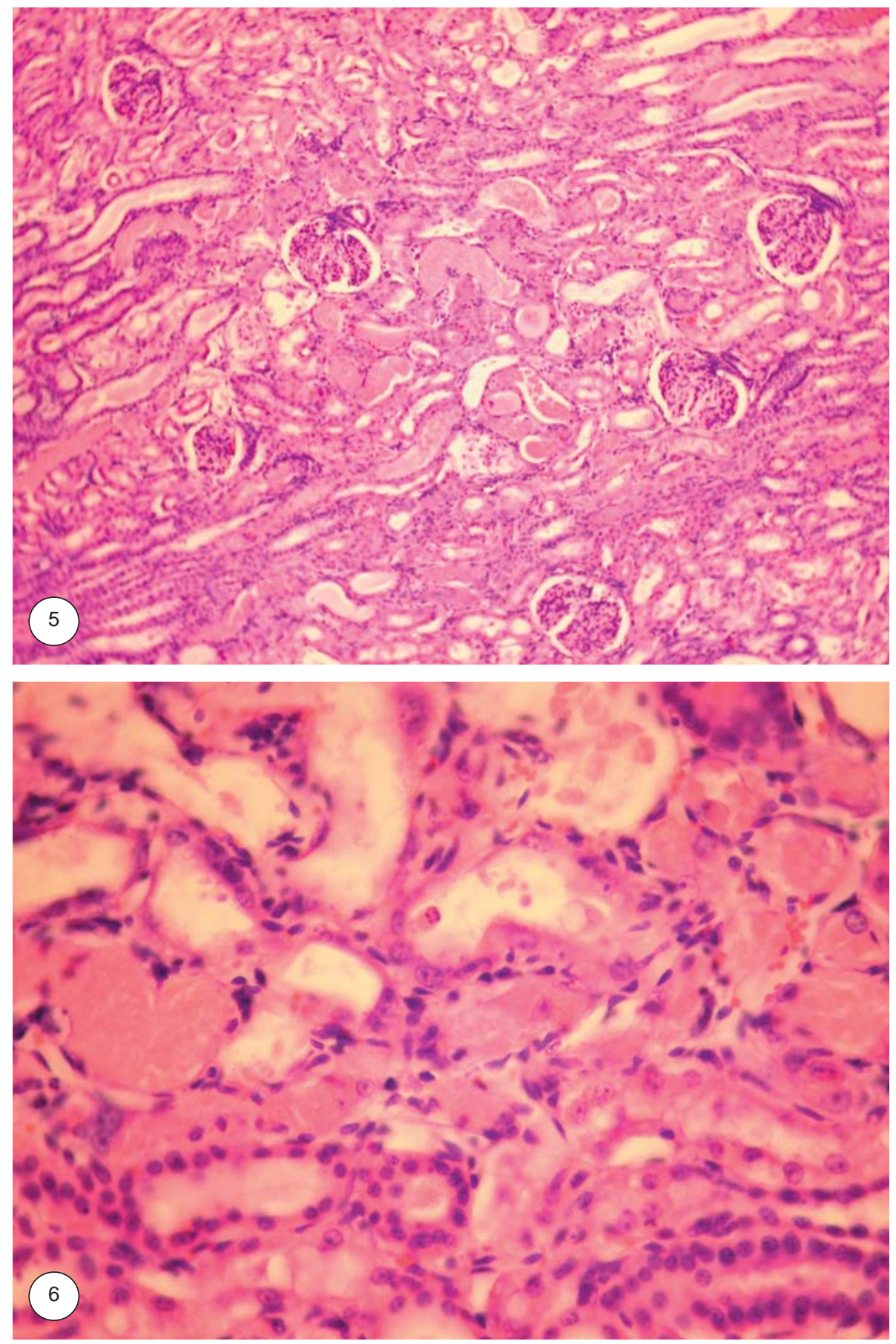

FIG. 5 and 6 Widespread necrosis of renal tubular epithelium, particularly in the proximal convoluted tubules. The tubules contain large numbers of protein casts in the lumens

ly affected. Epithelial cells showed karyolysis, karyorrhexis or pyknosis of nuclei with clumping and dissociation of the brightly eosinophilic cytoplasm (necrosis), or increased eosinophilia and vacuolated appearance (hydropic degeneration) of the cytoplasm. The tubules contained large numbers of protein casts in the lumens (Fig. 5 and 6). Single neu- trophils were present in the interstitial tissue and a mild neutrophilia was evident in the blood vessels. In one animal (no. 13) a few tubules were dilated and single tubules exhibited moderately basophilic epithelial cells with large, vesicular nuclei indicative of early regeneration. No specific lesions were visible in the glomeruli. 

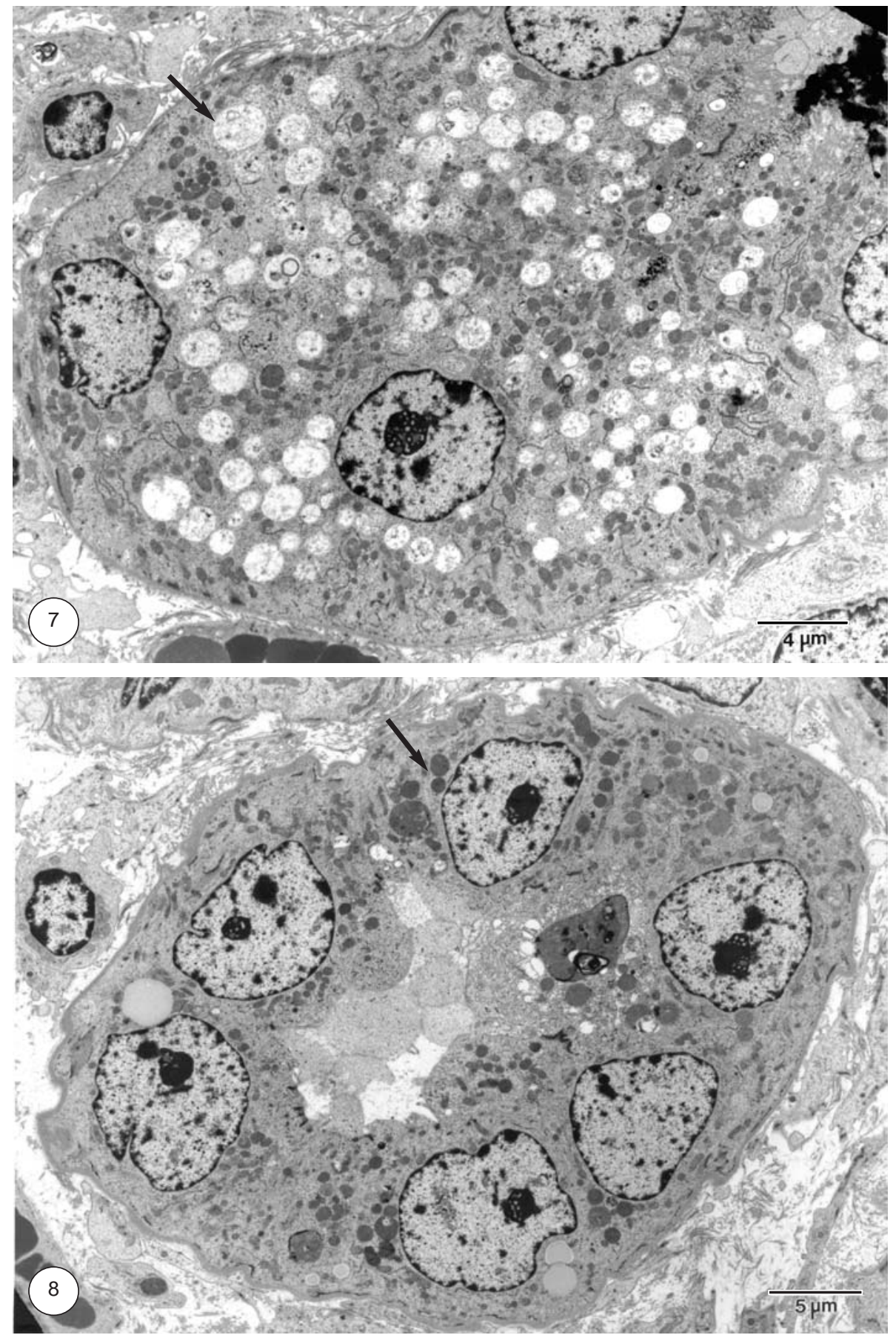

FIG. 7 Proximal convoluted tubule epithelial cell. Note the swollen mitochondria with loss of internal structure ( $\uparrow$ ). Parallel orientation of mitochondria to the cell axis is lost

FIG. 8 Proximal convoluted tubule epithelial cell. Note the increase in intracellular electrondense bodies interpreted as primary lysosomes ( $\uparrow$ )

\section{LIVER}

Mild hepatocellular swelling with accumulation of multiple small vacuoles in the cytoplasm of hepatocytes (indicating hydropic degeneration) was the most striking lesion in most animals. Scattered hepa- tocytes throughout the parenchyma were necrotic, evidenced by increased eosinophilia of the cytoplasm and karyorrhexis, karyolysis or pyknosis of the nuclei. No specific lesions were visible in the portal tracts and bile ducts. 

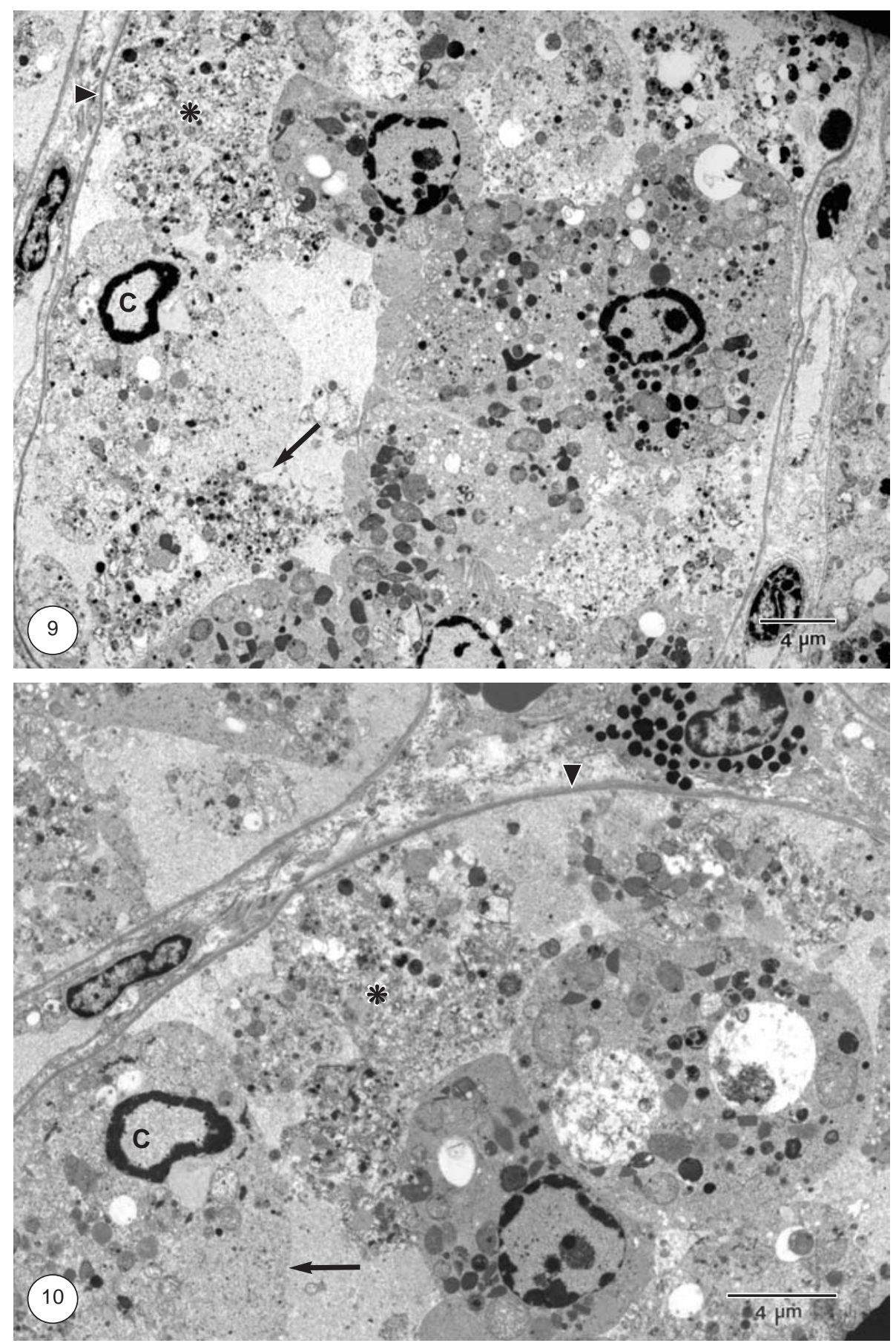

FIG. 9 and 10 Proximal convoluted tubule epithelium. Note the loss of cell membranes $(\boldsymbol{\uparrow})$ with chromatin margination (C). Cytoplasmic organelles are disorganized and dispersed as a loose mass within the tubular lumen(*). The basal membrane is intact $(\boldsymbol{\nabla})$

\section{Transmission electron microscopy}

\section{KIDNEY}

The most striking changes occurred in the PCT of both animals (Fig. 7-10). Individual cells lining the tubules could not be identified because of loss of the cell membranes. Affected tubules were demarcated by the intact basement membrane. The usually conspicuous brush border and apical canaliculi of the epithelial cells had disappeared. The cytoplasmic organelles and nuclei were disorganized and dispersed as a loose mass within the tubular lumen. 
Nuclear changes ranged from chromatin margination and karyolysis to pyknosis. Chromatin margination, the most common change exhibited, was evident as condensation of the chromatin in irregular clumps (karyorrhexis), or as a thin continuous rim along the inner membrane of the nuclear envelope, with disappearance of the chromatin from other areas of the nucleus and loss of nucleolus. Some affected nuclei were interpreted as being in an intermediate stage between margination and lysis. Pyknotic nuclei were recognized by the shrunken nucleus with diffuse condensation of the chromatin.

The most conspicuous change in the proximal tubular epithelial cell cytoplasm was increased numbers of electron-dense, single membrane-bound bodies interpreted as primary lysosomes. Also present were variably sized, single membrane-bound vacuolar bodies which contained medium electron-dense, finely granular material that could not be identified because of the advanced stage of breakdown and degradation and most probably represented autolysosomes. Scattered between the necrotic cellular organelles were residual bodies loaded with undigested electron-dense residues of various kinds.

The normal basal distribution of the mitochondria, with parallel orientation to the cell axis, was lost. Mitochondria had a rounded form and some were severely swollen with a loss of internal structure, whereas others were electron-dense and could not be distinguished unequivocally, by morphological features alone, from other vesicular structures in the cell including primary lysosomes.

\section{DISCUSSION}

Following ingestion of $N$. gariepina there appears to be a threshold before toxicity will be induced. When $3 \mathrm{~g} / \mathrm{kg}$ of dried, milled plant material was dosed orally, five of the seven sheep died acutely or were euthanased for humane reasons. On the other hand, administration of $1.5 \mathrm{~g} / \mathrm{kg}$ elicited no clinical signs and $2.25 \mathrm{~g} / \mathrm{kg}$ only induced mild clinical signs. During the reported field outbreaks, the cattle were deprived of feed for 24-48 $\mathrm{h}$ and, following their release, they presumably consumed large quantities of the plant material (Du Plessis 2004; Du Plessis et al. 2004), thus most probably exceeding the threshold level.

Although this plant is highly toxic it has, except for the outbreak in cattle, never before been associated with poisoning of livestock (Du Plessis 2004). The acute mortalities observed in this study could be ascribed to other toxins, besides the nephrotox- in(s), contained by the plant. Analysis of the plant material for cyanogenic potential gave negative results. The nephrotic syndrome corroborated by the gross and histological findings was only reproduced in sheep that survived for longer than $24 \mathrm{~h}$.

The concentrations of non-protein nitrogen substances in the plasma generally increase when GFR decreases by more than $75 \%$ (more than $75 \%$ kidney damage has occurred). This is because of the large "reserve capacity" of the kidneys (Rose \& Renkke 1994). In the animals, which did not die acutely, kidney damage was so severe, enough to induce marked increases in the plasma concentrations of creatinine and urea as well as a low GFR. As no specific damage to the glomerulus was described on histopathology and there is no reason to believe that the azotaemia was pre-renal (the animals were not dehydrated, neither was any cardiac pathology detected), the drastic fall in GFR was due to blockage of proximal tubules by cellular debris and protein casts (as was obvious on the histopathological findings). The reverse pressure across the filtration membrane due to such blockage would effectively stop the filtration process.

The appearance of sodium in the urine and the rise of potassium concentration in the urine, as well as the increase in urine volume, despite the marked fall in GFR, could be ascribed to two factors. The flow of filtrate in non-damaged nephrons may have been so rapid that there was little chance for the reabsorption of sodium, potassium and water. More likely, however, in damaged nephrons (proximal tubular epithelial damage with loss of brush border), which still permitted filtrate flow, sodium, potassium and water reabsorption would be compromised. Most of the sodium, potassium and water reabsorption occurs in the PCT and damage to the renal epithelium in this region would account for losses of large amounts of electrolytes and water (by osmotic diuresis) (Knepper \& Burg 1983).

The reduction in urine osmolarity indicates, that, in spite of the natriuresis and kaliuresis, more water relative to electrolytes was lost. This may be a phenomenon associated purely with proximal tubular damage or possibly the distal part of the nephron duct becomes refractory to the effects of antidiuretic hormone (as in chronic nephritis).

Gamma glutamyltransferase is present in the brush border of the proximal convoluted tubule where it facilitates transfer of glutamate into the tubular cells (Welbourne \& Mathews 1999). A rise in urine GGT activities when there is proximal tubular damage, is 
therefore in accordance with the pathological findings in this section of the nephron tubule, as was seen in this trial.

The pathological lesions in the experimental sheep correspond to a large extent with the lesions described in the experimentally produced cases in cattle, but were much less pronounced compared to the lesions in the field outbreak in cattle (Du Plessis 2004). In both species under experimental conditions gross lesions included mild to moderate accumulation of fluid in body cavities, severe nephrosis and a moderate hepatosis. Contrary to the situation in the experimentally produced cases in cattle, perirenal oedema was not a striking feature in sheep, whereas widespread serosal haemorrhages were present in the latter.

Nephrosis was characterized by degeneration and necrosis of particularly the PCT and affected tubules contained large numbers of protein casts in the lumens. The mild neutrophyllic infiltration in the interstitial tissue was most likely in response to the tubular necrosis. Scattered tubules were dilated and in one animal early tubular epithelial regeneration was displayed. Transmission electron microscopy confirmed that the basement membrane of affected tubules was still intact and a significant increase of autolysosomes was displayed in the affected lining epithelial cells of the PCT. No structural visible lesions were evident in the glomeruli. Most animals revealed mild hepatocellular swelling, with accumulation of multiple small vacuoles in the cytoplasm of hepatocytes indicating hydropic degeneration.

The PCT epithelium is the most susceptible to both toxic and hypoxic injury because of its high metabolic rate (Jamison, Myers \& Neild 1997; Confer \& Panciera 2001). Degeneration and necrosis of the tubular epithelium in the kidneys (nephrosis), especially the PCT, is a non-specific lesion. It can be hypoxic or nephrotoxic in origin. The former follows on a period of hypotension causing marked renal ischaemia. Complete ischaemia of more than $2 \mathrm{~h}$ duration causes irreversible tubular necrosis, especially of the PCT (Confer \& Panciera 2001).

Nephrotoxic acute tubular necrosis (ATN) can be distinguished from ischaemic ATN by two histological features viz. extensive necrosis of predominantly PCT epithelium and intact tubular basement membranes. The opposite is true for ischaemic ATN, where necrosis is patchy and mostly affects the PCT with disruption of the basement membrane (Maxie 1993; Jamison et al. 1997; Confer \& Panciera 2001).
Toxic damage to tubular epithelium has a better prognosis if the animal survives the initial toxic insult, as the basement membranes that are necessary for epithelial regeneration are intact (Maxie 1993), and some nephrotoxic drugs can cause ischaemia rather than direct toxic damage to the renal tubules (Jamison et al. 1997).

In conclusion, dosing of $N$. gariepina plant material to sheep in doses exceeding $2.8 \mathrm{~g} / \mathrm{kg}$ resulted in acute death of most animals and marked changes in the clinical chemistry of the survivors characterized by a sudden, marked decrease in GFR, azotaemia, elevated fractional excretion of $\mathrm{Na}$ and $\mathrm{K}$, and increased urinary GGT activity.

Sheep, which died or were euthanased, exhibited severe nephrosis that was histologically characterized by necrosis of predominantly lining epithelial cells of the PCT and the presence of protein and cellular casts.

\section{ACKNOWLEDGEMENTS}

We thank Ms H. Engelbrecht and Ms H. Rossouw, Section of Physiology; Ms E. Myburgh, Section of Clinical Pathology; Ms E. van Wilpe and Ms D. Meyer, Electron Microscopy Unit, Faculty of Veterinary Science, University of Pretoria; and Ms L. Labuschagne, Division of Toxicology, Onderstepoort Veterinary Institute for their valuable assistance. The authors also express their gratitude to the National Research Foundation (NRF) of South Africa and Mr W. du Plessis, the farmer, for funding this trial. The research reported here emanates from Project 36.5.520, approved by the Animal Use and Care and Research Committees of the Faculty of Veterinary Science, University of Pretoria.

\section{REFERENCES}

BASTL, C.P., RUDNICK, M.R. \& NAIRNS, R.G. 1985. Assessment of renal function: characteristics of the functional and organic forms of acute renal failure, in The kidney: physiology and pathophysiology, edited by D.W. Seldin \& G. Giebisch. New York: New York Press.

CONFER, A.W. \& PANCIERA, R.J. 2001. The urinary system, in Thomson's Special Veterinary Pathology, $3^{\text {rd }}$ ed., edited by M.D. McGavin, W.W. Carlton \& J.F. Zachary. Missouri: Mosby Inc.

DU PLESSIS, E.C. 2004. Pathological investigation of the nephrotoxic effects of the shrub Nolletia gariepina (DC) Mattf. in cattle. MMedVet (Path) dissertation, University of Pretoria.

DU PLESSIS, E.C., JOUBERT, J.P.J., PROZESKY, L., NAUDÉ T.W., HERMAN, P. \& VAN DER WESTHUIZEN, G.B.M. 2004. Nephrotic syndrome in cattle caused by the shrub Nolletia gariepina in the Kalahari sandveld of southern Africa, in Poisonous Plants and Related Toxins, edited by T. 
Acamovic, C.S. Stewart \& T.W. Pennycott. Wallingford: CABI Publishing.

JAMISON, R.L., MYERS, B.D. \& NEILD, G. 1997. Acute renal failure, in Nephrology, edited by R.L. Jamison \& R. Wilkinson. London: Chapman \& Hall Medical.

KNEPPER, M. \& BURG, M. 1983. Organisation of nephron function. American Journal of Physiology, 244, F579-F589.

MAXIE, M.G. 1993. The urinary system, in Pathology of domestic animals, $4^{\text {th }}$ ed., edited by K.V.F. Jubb, P.C. Kennedy \& N. Palmer. California: Academic Press Inc.
NAWAZ, M. \& SHAH, B.H. 1984. Renal clearance of endogenous creatinine and urea in sheep during summer and winter. Research in Veterinary Science, 36:220-224.

ROSE, B.D. \& RENKKE, H.G. 1994. Review of renal physiology, in Renal pathophysiology - the essentials. Baltimore: Williams \& Wilkins.

WELBOURNE, T.Z. \& MATHEWS, J.C. 1999. Glutamate transport and renal function. American Journal of Physiology, 277 (4 Pt2), F501-F505. 